\title{
Efficacy of umbilical cord-derived mesenchymal stem cell-based therapy for osteonecrosis of the femoral head: A three-year follow-up study
}

\author{
CHUN CHEN ${ }^{1,2}$, ZHIGUO QU ${ }^{3}$, XIAOGUANG YIN ${ }^{2}$, CHUNYU SHANG $^{1}$, QIANG AO ${ }^{4}$, \\ YONGQUAN GU ${ }^{5}$ and YING $\mathrm{LIU}^{2,6}$ \\ ${ }^{1}$ Department of Vascular Interventional Surgery, Siping Hospital of China Medical University; \\ ${ }^{2}$ Tuhua Bioengineering Company Ltd.; ${ }^{3}$ Department of Orthopaedic Surgery, Siping Hospital of \\ China Medical University, Siping, Jilin 136000; ${ }^{4}$ Department of Tissue Engineering, China Medical University, \\ Shenyang, Liaoning 110122; ${ }^{5}$ Department of Vascular Interventional Surgery, Xuanwu Hospital of \\ Capital Medical University, Beijing 100053; ${ }^{6}$ The Key Tissue Engineering Laboratory \\ of Jilin Province, Siping, Jilin 136000, P.R. China
}

Received March 9, 2016; Accepted August 30, 2016

DOI: $10.3892 / \mathrm{mmr} .2016 .5745$

\begin{abstract}
This is a retrospective analysis of the clinical effects of transplant of mesenchymal stem cells (MSCs) derived from human umbilical cord-derived MSCs (hUC-MSCs) for the treatment of osteonecrosis of the femoral head (ONFH). The biological characteristics of hUC-MSCs were assessed using flow cytometry. Nine eligible patients were enrolled in the study as they adhered to the Association Research Circulation Osseous (ARCO) classification of stage II-IIIa, and hUC-MSCs were grafted by intra-arterial infusion. Organize effective perfusion was assessed using the oxygen delivery index (ODI). The results showed that the ODI was increased at three days post-operation. The MRI results revealed that at 12 and 24 months after treatment, the necrotic volume of the femoral heads was significantly reduced. No obvious abnormalities were observed. Taken together, these data indicate that intra-arterially infused hUC-MSCs migrate into the necrotic field of femoral heads and differentiate into osteoblasts, thus improving the necrosis of femoral heads. This finding suggested that intra-arterial infusion of hUC-MSCs MSCs is a feasible and relatively safe method for the treatment of femoral head necrosis.
\end{abstract}

Correspondence to: Professor Ying Liu, The Key Tissue Engineering Laboratory of Jilin Province, 89 Nanyingbin Road, Siping, Jilin 136000, P.R. China

E-mail: 1y3641829@163.com

Dr Yongquan Gu, Department of Vascular Interventional Surgery, Xuanwu Hospital of Capital Medical University, 45 Changchun Street, Beijing 100053, P.R. China

E-mail: yongquan_gu@126.com

Key words: mesenchymal stem cell, osteonecrosis of the femoral head, therapy

\section{Introduction}

Osteonecrosis of the femoral head (ONFH) is a type of common and refractory disease $(1,2)$. Young adults primarily suffer from ONFH and the long-term results of total hip arthroplasty (THA) are usually unpredictable in this age group $(3,4)$. A variety of surgical procedures have been used to maintain the femoral head and avoid THA in younger patients. These approaches include core decompression $(5,6)$, and various types of osteotomies (7). Nevertheless, despite these advances, the optimal treatment for patients with ONFH in clinical practice is not yet defined.

Previous findings have demonstrated the efficacy of mesenchymal stem cells (MSCs) implanted into the femoral head for the treatment of ONFH $(8,9)$. MSCs can be isolated from bone marrow and adipose tissues in adult stages and from umbilical cord (UC) blood, and connective tissue (Wharton's jelly) of human UC (10-13). MSCs can differentiate into specialized cells to repair injured tissues, under certain conditions based on their potential capacity of multidirectional differentiations $(14,15)$. Thus, stem cell transplantation has gradually emerged as a promising approach for the treatment of ONFH $(8,16,17)$. In previous studies, we grafted human UC-derived MSCs (hUC-MSCs) to treat non-union in rats and human (11-13). Our results demonstrated the safety and efficacy of osteoblastic differentiation of hUC-MSCs. However, to the best of our knowledge, few studies are available regarding the fate and distribution of hUC-MSCs in the treatment of ONFH. Furthermore, the delivery approaches are a wide concern and unsolved problem.

In the present study, we investigated wether hUC-MSCs are useful in treating ONFH. As ONFH is primarily caused by a partial obstruction of the blood supply to the femoral head, and aggrevated by insufficient blood supply to trabecular bone and bone cell death in the femoral head; we grafted hUC-MSCs by intra-arterial infusion. We hypothesized that: i) the implanted hUC-MSCs could differentiate into endothelial cells, which in 
turn were able to regenerate blood vessels supplying the femoral head or produce more collateral circulation; and ii) differentiate into osteoblasts and reconstruct the necrotic area in femoral head. In the present study, we retrospectively reported 9 patients with ONFH who accepted intra-arterial infusion of hUC-MSCs treatment. The clinical and radiographic outcomes in the subsequent 24 months were evaluated.

\section{Materials and methods}

Eligibility criteria. This single-center randomized clinical trial was conducted in the Siping Hospital of China Medical University (Jilin, China) between January, 2011 and January, 2014. The aim of the present study was to assess the efficacy of hUC-MSCs grafting by intra-arterial infusion for the treatment of early-stage ONFH. The protocol of the present study was approved by the Institutional Review Board and the Ethics Committee of Siping Hospital of China Medical University. Written informed consent was obtained from each patient before enrollment.

Study design. Patients were evaluated clinically and radiologically using the Association Research Circulation Osseous (ARCO) classification (18) and the Harris hip score (HHS) (19) pre-operatively and at 12 and 24 months post-operation. All the patients in this study were $\geq 18$ years of age, and were classified as ARCO stages II to IIIa. The patients had femoral head collapse of $<2 \mathrm{~mm}$ and no evidence any damage to the acetabular cartilage. Those patients who were excluded from this study had damages caused by a traumatic event or experienced severe hemorrhage. We also excluded those who were not available for follow up because of life-threatening conditions or had missing stratification information. Those without standard care and rehabilitation were also excluded. This exclusion criterion was chosen to ensure that all the patients were treated according to the best medical practice.

We included 9 patients (9 hips) for this study. The general characteristics of the patients are shown in Table I. Blood was taken from the internal jugular veins. The outcomes were evaluated based on the HHS and the radiological results.

Harvesting of UC. Six human equally sized UC were collected after informed consent was obtained from mothers in accordance with the Ethics Committee of the Institute of Siping Hospital of China Medical University. Informed consent was obtained from all the cases. Experiments and laboratory procedures were carried out in the Siping Hospital of China Medical University. From each sample, sections of $8-10 \mathrm{~cm}$ of the UCs were internally washed with phosphate-buffered saline (PBS) containing $300 \mathrm{U} / \mathrm{ml}$ penicillin and $0.3 \mathrm{mg} / \mathrm{ml}$ streptomycin and immediately immersed in Dulbecco's modified Eagle's medium-low glucose (DMEM-LG) supplemented with 10\% AB-human serum (all from Gibco, Grand Island, NY, USA), 300 U/ml penicillin, and $0.3 \mathrm{mg} / \mathrm{ml}$ streptomycin. The samples were processed within $12-15 \mathrm{~h}$ after collection.

Isolation and culture of adherent cells from UC (11-13). UCs were filled with $0.1 \%$ collagenase (Sigma-Aldrich, St. Louis,
MO, USA) in PBS and incubated at $37^{\circ} \mathrm{C}$ for $20 \mathrm{~min}$. Each $\mathrm{UC}$ was washed with proliferation medium (a-MEM $+10 \%$ AB-human serum), and the detached cells were harvested after gentle massage of the UC. The cells were centrifuged at $300 \mathrm{x} \mathrm{g}$ for $10 \mathrm{~min}$, resuspended in proliferation medium, and seeded in $75-\mathrm{cm}^{2}$ flasks at a density of $5 \times 10^{7}$ cells $/ \mathrm{ml}$. After $24 \mathrm{~h}$ of incubation, non-adherent cells were removed, and culture medium was replaced every 3 days. Adherent cells were cultured until they reached $80-90 \%$ confluence. The cells were observed using a phase contrast microscope (Olympus, Tokyo, Japan).

Flow cytometry. To analyze the cell-surface expression of typical protein markers, adherent cells were passaged with $0.25 \%$ trypsin (Gibco). The cells were washed in PBS and fixed in a $4 \%$ paraformaldehyde solution (both from Sigma-Aldrich, Milwaukee, WI, USA) for $10 \mathrm{~min}$; and incubated with the following anti-human primary antibodies: mouse monoclonal CD45-phycoerythrin (PE) (Abcam, Cambridge, MA, USA; catalog no.: ab25603; dilution: $0.2 \mu \mathrm{g} / 10^{6}$ cells), mouse monoclonal CD31-fluorescein isothiocyanate (FITC) (Abcam; catalog no.: ab33858; dilution: $10 \mu 1 / 10^{6}$ cells), mouse monoclonal CD90-FITC (Abcam; catalog no.: ab11155; dilution: $10 \mu 1 / 10^{6}$ cells), mouse monoclonal HLA-DR-PE (Abcam; catalog no.: ab95830; dilution: $5 \mu \mathrm{l} / 10^{6}$ cells). A total of 10,000 labeled cells were analyzed using a Guava easyCyte flow cytometer running Guava Express Plus software (Guava Technologies, Inc., Chicago, IL, USA).

hUC-MSC osteogenic differentiation. For osteocyte differentiation, the cells were plated at $10^{4}$ cells $/ \mathrm{cm}^{2}$ on uncoated plastic (Permanox) chamber slides (Lab-Tek; Thermo Fisher Scientific, Inc., Waltham, MA, USA) and allowed to adhere for $24 \mathrm{~h}$ in tissue culture media (DMEM-LG and 10\% human AB serum), after which the media were replaced with osteogenic induction media, composed of DMEM-LG supplemented with $10 \%$ human $\mathrm{AB}$ serum, $1 \%$ penicillin/streptomycin, $50 \mu \mathrm{g} / \mathrm{ml}$ L-ascorbic acid (Wako Chemicals GmbH, Neuss, Germany), $10 \mathrm{mM}$ glycerol phosphate disodium salt ( $\beta$-glycerophosphate), $10 \mathrm{nM}$ dexamethasone, and $10 \mathrm{nM}$ calcitriol (1 $\alpha, 25$-dihydroxyvitamin D3) (Sigma, Irvine, UK).

hUC-MSC osteogenic mineralization. Alizarin Red S (ARS) staining was used to examine osteogenic differentiation and mineralization. Specimens were washed with PBS, fixed with $10 \%$ formaldehyde, and stained with ARS (Millipore Corp., Billerica, MA, USA), which stained calcium minerals into a red color.

Red blood cell related parameters measurements. Red blood cell-related parameters were analyzed as blood cells before operation and third day after operation using an automated blood cell counter LH-750 (Beckman Coulter, Inc., Brea, CA, USA).

Analysis of magnetic resonance imaging (MRI). MRI was performed using Discovery MR750 3.0T (GE Medical Systems, Milwaukee, WI, USA) before and 12/24 months after intra-arterial infusion with coronal T1-weighted imaging, T2-weighted imaging and axial T1-weighted imaging, and 
T2-weighted imaging. Images (3-mm) with a $0.5-\mathrm{mm}$ gap were obtained using a 256x192 matrix and four excitations. The volumetric analysis of the ONFH was assayed by Syngo via 2.0 (GE Medical Systems, Milwaukee, WI, USA).

Surgical procedure. Under general anesthesia, each patient was placed in the supine position, and the right femoral artery was punctured using the Seldinger technique with a $4.0 \mathrm{~F}$ Cobra catheter (Terumo, Tokyo, Japan). MSCs (10 ml) with a cell density of $5 \times 10^{6}$ to $1 \times 10^{7} / \mathrm{ml}$ were intra-arterially injected. Once the needle was fully withdrawn, the puncture site was wrapped with sterilized dressing. The patients remained in the supine decubitus on the operation bed for another $30 \mathrm{~min}$ before being returned to individual wards. Antibiotics were given to prevent infection.

The patients were instructed to be non-weight-bearing for 4 weeks and partial weight-bearing for the subsequent 6 weeks. Full weight-bearing was achieved 6 months postoperatively (20).

Oxygen delivery index (ODI). ODI was calculated as the ratio between the hematocrit and systolic blood viscosity $(\mathrm{SBV})(21,22) . \mathrm{ODI}=$ hematocrit $/ \mathrm{SBV}=100 \mathrm{H} / 1.4175+5.87$ $8 \mathrm{H}-12.98 \mathrm{H}^{2}+31.964 \mathrm{H}^{3}$, where $\mathrm{H}$ is the volume fraction of erythrocytes.

Treatment protocol. The following treatment protocol was used: i) anti-platelet aggregation drugs: sarpogrelate (Mitsubishi Tanabe Pharma Corp, Tianjin, China) $100 \mathrm{mg}$ t.i.d. + clopidogrel hydrogen sulfate (Salubris Co., Ltd., Shenzhen, China) $50 \mathrm{mg} / \mathrm{day}$. According to the target lesions run-off situation, dual anti-platelet drugs should continued one year post-operation at least; ii) medications for improving circulation: alprostadil injection (Tide Pharmaceutical Co., Ltd., Beijing, China); and iii) perioperative anticoagulation drugs. Low molecular weight heparin sodium injection 4000 IU q12h hypodermic injection (Clexane; Aventis Intercontinental, Antony, France) was also used.

Evaluation and statistical analysis. The evaluations consisted of clinical and radiographic analysis preoperatively and at the end of follow-up based on the HHS. All the patients underwent clinical and radiographic examinations at 12 and 24 months post-operation. An excellent or good HHS was defined as $\geq 80$ points and scores $<70$ were considered negative. Analyses were completed using SPSS version 22.0 software (IBM SPSS, New York, NY, USA).

General characteristics of the ONFH patients. The general characteristics of patients are shown in Table I. The patients included 4 males and 5 females, with an age range of 28-51 years (mean, $41.13 \pm 3.29$ years). The etiology of the osteonecrosis was corticosteroid use in 6 hips, intemperance in 2 hips, and idiopathic or unknown in 1 hips; 5 hips had stage II osteonecrosis and 4 had stage IIIa osteonecrosis.

\section{Results}

Evaluation of hUC-MSCs. Cells derived from UC were observed $24 \mathrm{~h}$ after they were seeded (Fig. 1A), when part of
Table I. General characteristics of ONFH patients.

Characteristics

Data

Gender (no. of pts.)

Male

4

Female

Invasive hip (no. of pts.)

Unilateral

Bilateral

Etiology (no. of pts.)

Idiopathic

Corticosteroids

Alcohol

Trauma

ARCO staging (no. of hips)

II

IIIa 4

ONFH, osteonecrosis of the femoral head; ACRO, Association Research Circulation Osseous.

the round mononuclear cells was adherent. Three days after inoculation, small colonies of the adherent cells with typical fibroblast-shaped morphology were obtained (Fig. 1B). These primary cells reached monolayer confluence, after planting for 5-6 days, when passaged for the first time. Fifth-passaged cells were analyzed by flow cytometry, and were strongly positive for CD31 (98.95\%) and CD90 (97.77\%), but negative for CD45 (0.55\%) and HLA-DR (0.14\%) (Fig. 1C and D).

hUC-MSC osteogenic mineralization. ARS staining was used to examine osteogenic mineralization. The purpose of this method was to confirm that hUC-MSCs cultured in osteogenic media were able to produce minerals. ARS stained calcium minerals into a red color (Fig. 2), while no red staining was found in hUC-MSCs cultured in tissue culture media, which served as a control (data not shown). hUC-MSCs in osteogenic media showed significant mineral staining from the 7 th day (Fig. 2). Red staining became progressively thicker and darker at 14 and 21 days.

Patient-specific extent-related parameters of red blood cells and platelet data. The pre-operation red cell count, $\mathrm{Hb}$ and Hct levels were significantly reduced three days after the operation. The same results were observed in the mean cell volume, mean cell $\mathrm{Hb}$ concentration, mean cell $\mathrm{Hb}$ and red cell distribution width. Related parameters of platelet remained unchanged at all time points, as anti-platelet aggregation drugs were used before and after the operation (Table II).

ODI changes. As shown in Table III, after operation, ODI in all the patients increased by about $5 \%$ compared to pre-operation readings. This increase was especially more obvious in the ARCO stage II patients. These results consisted of Hct reduction, which suggested a more efficient oxygen transport (23). 
$\mathbf{A}$

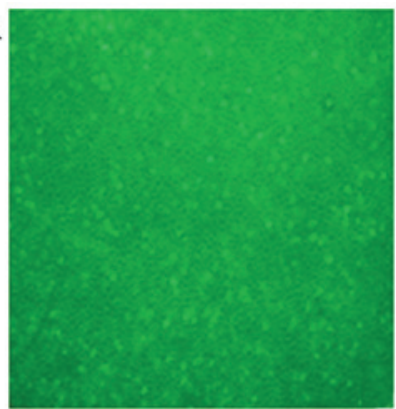

C

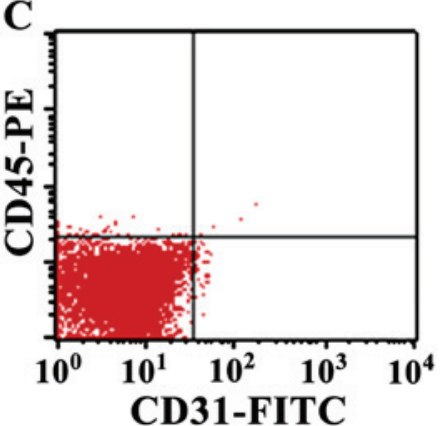

B

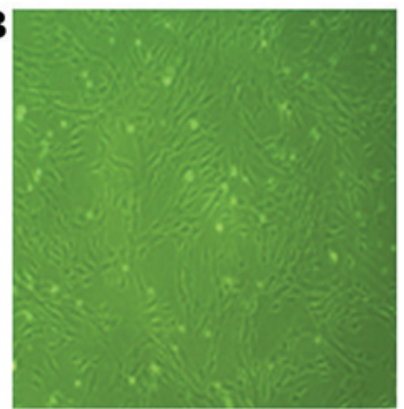

D

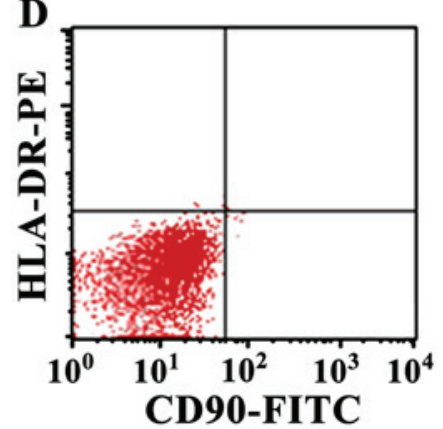

Figure 1. The characteristics of HUC-MSCs. (A) The cells derived from UC were observed $24 \mathrm{~h}$ after they were seeded. (B) Fifth-passaged cells show typical fibroblast-shaped morphology. (C) Fifth-passaged cells were analysis of CD31 and CD45 by flow cytometry. (D) Fifth-passaged cells were analysis of CD90 and HLA-DR by flow cytometry. hUC-MSCs, human umbilical cord-derived MSCs; UC, umbilical cord.

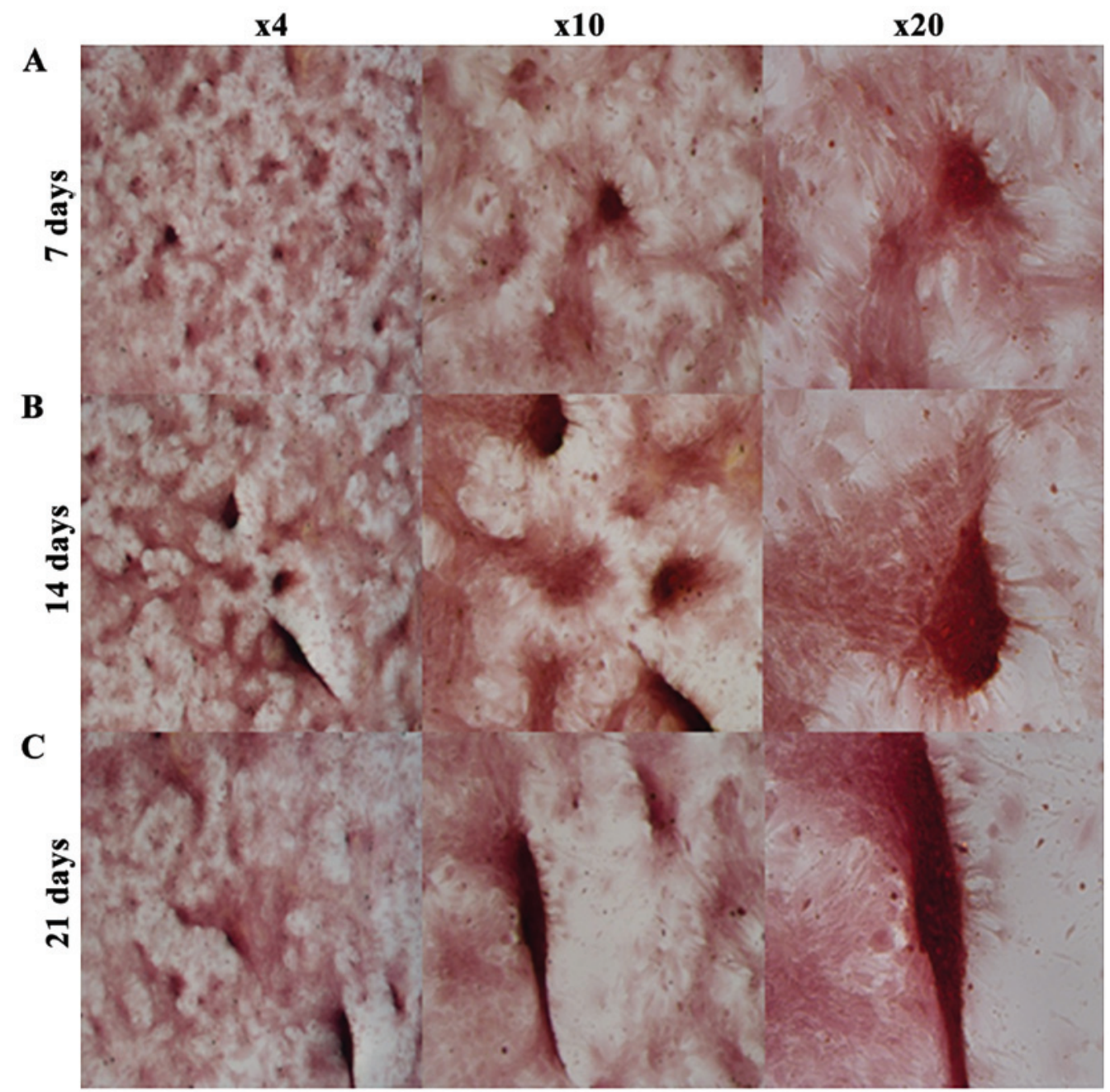

Figure 2. ARS staining of bone mineralization by hUC-MSCs. It stains calcium minerals into a red color. (A) hUC-MSCs cultured in osteogenic media at 7 days . (B) hUC-MSCs cultured in osteogenic media at 14 days. (C) hUC-MSCs cultured in osteogenic media at 21 days. ARS, Alizarin Red S; hUC-MSCs, human umbilical cord-derived MSCs. 


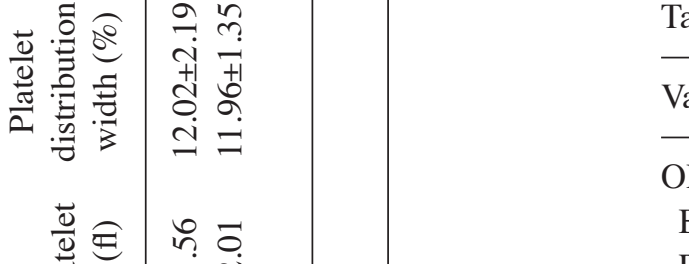

Table III. ODI changes after operation in 3 days.

\begin{tabular}{ccc}
\hline Variables & ARCO stage II & ARCO stage IIIa \\
\hline ODI & & \\
BO & $10.31 \pm 0.26$ & $10.03 \pm 0.27$ \\
PO & $10.86 \pm 0.34^{\mathrm{a}}$ & $10.53 \pm 0.09^{\mathrm{a}}$
\end{tabular}

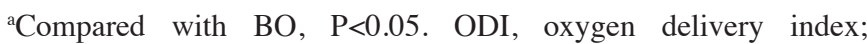
ARCO, Association Research Circulation Osseous.

Regression of a necrotic lesion after intra-arterial infusion on MRI. MRI was performed to determine conditions of $\mathrm{ONFH}$ after 12 months. The patients showed sporadic low signal intensity on T1W, but no obvious abnormal signal intensity on T2W $\mathrm{R}$, profile rules, and comparatively smooth edge (Fig. 3B). The result from the volumetric analysis showed that the necrotic volume of femoral heads was $7.16 \pm 0.73 \mathrm{~cm}^{3}$; whereas, the necrosis volume at 24 months decreased to $5.86 \pm 1.67 \mathrm{~cm}^{3}$ $(\mathrm{p}<0.05)$ (Fig. 3C).

HHS evaluation. The mean preoperative HSS was $39.19 \pm 5.06$ points pre-operation (Table IV). HHS points increased obviously at the end of the 12 month postoperation, but these points decreased $10 \%$ by the end of the 24 -month post-operation.

\section{Discussion}

We treated the ONFH patients with hUC-MSCs by intra-arterial infusion, and assessed the efficacy ischemia reperfusion with ODI in the acute phase postoperative and imaging evaluation in the following 24 months. To the best of our knowledge, this is the first study on the subject of predicting the efficacy of ONFH treatment with ODI index. We also showed that the migration of hUC-MSCs promoted bone formation in the ischemia area in ONFH patients. Our experimental results supported cell therapy for ONFH. The hUC-MSCs used in the current study meet the criterion of the International Society for Cell Therapy (24). The grafted hUC-MSCs differentiated into osteoblasts in vitro. In this experiment, we compared the postoperation red blood cell-related parameters with preoperative results and ODI value. The number of red blood cells and HCV values were all reduced postoperatively. This was advantageous to the red blood cells through newborn capillaries to carry oxygen. The HHS points increased by the end of the 3rd month post-operation. Two patients of ARCO II stage had over 80 HHS at the end of the 24th month. Additionally, sporadic low signal intensity on T1W, and no obvious abnormal signal intensity on T2W was observed by MRI scan at the end of 12 months, and comparatively smooth edge after 24 months of treatments. The rate of radiological progression on the necrotic lesion after hUC-MSCs treatment was statistically lower than pre-operation. In addition, the results from the volumetric analysis revealed the necrotic volume of femoral heads decreased. Therefore, we considered that intra-arterial infused hUC-MSCs in ONFH could migrate into the necrotic field of femoral head, multiply and differentiate into osteoblasts. Taken together, these results 

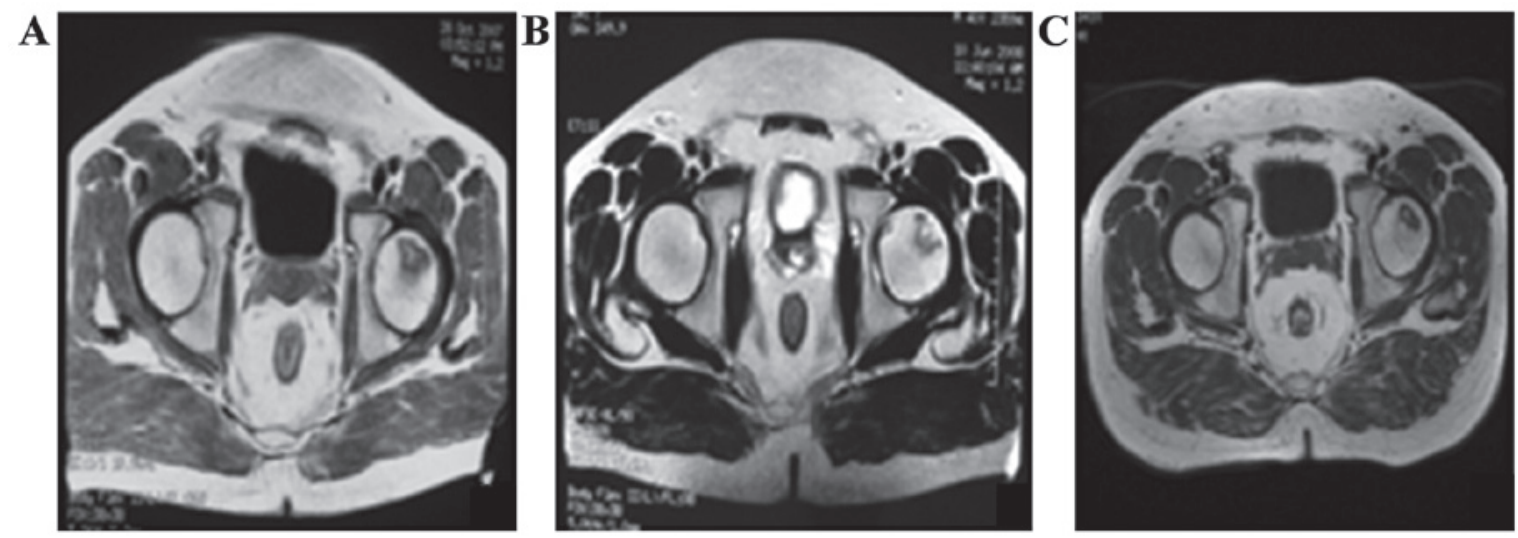

Figure 3. Regression of a necrotic lesion after intra-arterial infusion on MRI. (A) MRI scan pre-operation. (B) MRI scan 12 months postoperation. (C) MRI scan 24th months postoperation. MRI, magnetic resonance imaging.

Table IV. Clinical results of survival hips.

ARCO stage II

$\begin{array}{cc}39.19 \pm 5.06 & 33.25 \pm 6.37 \\ 89.46 \pm 9.11 & 83.13 \pm 11.66 \\ 5 & 3 \\ 5,100 & 3,75 \\ 80.09 \pm 10.16 & 71.52 \pm 9.23 \\ 4 & 2 \\ 4,80 & 2,50\end{array}$

ARCO stage IIIa

HHS (preoperative)

HHS (12 months postoperation)

Survival (12 months postoperation ), $\mathrm{n}$

EGR (12 months postoperation), n, \%

HHS (24 months postoperation)

Survival (24 months postoperation), $\mathrm{n}$

EGR (24 months postoperation), n, \% survival of the hip, further research should be considered to combine this method with core decompression. We did not supervise the histopathology in this study, and the potential mechanism underlying this approach requires further investigation.

Consequently, the results have shown that hUC-MSCs with intra-arterial infusion were inclined to commit to repair and regeneration in the condition of bone necrosis. An important mechanism may be to improve the abovementioned signs of ONFH in imaging. We considered hUC-MSCs with intra-arterial infusion a feasible and relatively safe avenue for the treatment of ONFH. In addition, the long-term safety of hUC-MSCs with intra-arterial infusion needs further evaluation.

\section{Acknowledgements}

This study has been supported by the National High Technology Research and Development Program of (863 Program), nos. 2011AA020101 and 2012A020905.

\section{References}

1. Kaushik AP, Das A and Cui Q: Osteonecrosis of the femoral head: an update in year 2012. World J Orthop 3: 49-57, 2012.

2. Wang C, Wang Y, Meng HY, Yuan XL, Xu XL, Wang AY, Guo QY, Peng J and Lu SB: Application of bone marrow mesenchymal stem cells to the treatment of osteonecrosis of the femoral head. Int J Clin Exp Med 8: 3127-3135, 2015. 
3. Ince A, Lermann J, Göbel S, Wollmerstedt N and Hendrich C: No increased stem subsidence after arthroplasty in young patients with femoral head osteonecrosis: 41 patients followed for 1-9 years. Acta Orthop 77: 866-870, 2006.

4. Kim YH, Choi Y and Kim JS: Cementless total hip arthroplasty with alumina-on-highly cross-linked polyethylene bearing in young patients with femoral head osteonecrosis. J Arthroplasty 26: 218-223, 2011.

5. Persiani P, De Cristo C, Graci J, Noia G, Gurzì M and Villani C: Stage-related results in treatment of hip osteonecrosis with core-decompression and autologous mesenchymal stem cells. Acta Orthop Belg 81: 406-412, 2015.

6. Tabatabaee RM, Saberi S, Parvizi J, Mortazavi SM and Farzan M Combining concentrated autologous bone marrow stem cells injection with core decompression improves outcome for patients with Early-Stage osteonecrosis of the femoral head: a comparative study. J Arthroplasty 30 (Suppl 9): 11-15, 2015.

7. Sonoda K, Yamamoto T, Motomura G, Nakashima Y, Yamaguchi R and Iwamoto Y: Outcome of transtrochanteric rotational osteotomy for posttraumatic osteonecrosis of the femoral head with a mean follow-up of 12.3 years. Arch Orthop Trauma Surg 135: 1257-1263, 2015.

8. Zhao D, Liu B, Wang B, Yang L, Xie H, Huang S, Zhang Y and Wei X: Autologous bone marrow mesenchymal stem cells associated with tantalum rod implantation and vascularized iliac grafting for the treatment of end-stage osteonecrosis of the femoral head. Biomed Res Int 2015: 240506, 2015.

9. Jin H, Xu T, Chen Q, Wu C, Wang P, Mao Q, Zhang S, Shen J and Tong P: The fate and distribution of autologous bone marrow mesenchymal stem cells with intra-arterial infusion in osteonecrosis of the femoral head in dogs. Stem Cells Int 2016: 8616143, 2016.

10. Park HW, Chang JW, Yang YS, Oh W, Hwang JH, Kim DG and Paek SH: The effect of donor-dependent administration of human umbilical cord blood-derived mesenchymal stem cells following focal cerebral ischemia in rats. Exp Neurobiol 24 358-365, 2015.

11. Qu Z, Guo S, Fang G, Cui Z and Liu Y: AKT pathway affects bone regeneration in nonunion treated with umbilical cord-derived mesenchymal stem cells. Cell Biochem Biophys, 2014.

12. Qu Z, Guo L, Fang G, Cui Z, Guo S and Liu Y: Biological characteristics and effect of human umbilical cord mesenchymal stem cells (hUC-MSCs) grafting with blood plasma on bone regeneration in rats. Cell Biochem Biophys 63: 171-181, 2012.

13. Qu Z, Fang G, Cui Z and Liu Y: Cell therapy for bone nonunion: a retrospective study. Minerva Med 106: 315-321, 2015.

14. Klimczak A and Kozlowska U: Mesenchymal stromal cells and tissue-specific progenitor cells: their role in tissue homeostasis. Stem Cells Int 2016: 4285215, 2016.

15. Mattar P and Bieback K: Comparing the immunomodulatory properties of bone marrow, adipose tissue, and birth-associated tissue mesenchymal stromal cells. Front Immunol 6: 560, 2015.

16. Zhao D, Cui D, Wang B, Tian F, Guo L, Yang L, Liu B and Yu X: Treatment of early stage osteonecrosis of the femoral head with autologous implantation of bone marrow-derived and cultured mesenchymal stem cells. Bone 50: 325-330, 2012.
17. Mao Q, Jin H, Liao F, Xiao L, Chen D and Tong P: The efficacy of targeted intraarterial delivery of concentrated autologous bone marrow containing mononuclear cells in the treatment of osteonecrosis of the femoral head: a five year follow-up study. Bone 57: 509-516, 2013

18. Association Research Circulation Osseous: Committee on terminology and classification. ARCO News 4: 41-46, 1992.

19. Harris WH: Traumatic arthritis of the hip after dislocation and acetabular fractures: treatment by mold arthroplasty. An end-result study using a new method of result evaluation. J Bone Joint Surg Am 51: 737-755, 1969.

20. Zhao D, Zhang Y, Wang W, Liu Y, Li Z, Wang B and Yu X: Tantalum rod implantation and vascularized iliac grafting for osteonecrosis of the femoral head. Orthopedics 36: 789-795, 2013.

21. Kameneva MV, Watach MJ and Borovetz HS: Gender difference in oxygen delivery index: Potential link to development of cardiovascular diseases. Appl Cardiopulm Pathophysiol 9: 382-387, 2000

22. Cho YI and Cho DJ: Hemorheology and microvascular disorders. Korean Circ J 41: 287-295, 2011.

23. Tsai AG, Hofmann A, Cabrales P and Intaglietta M: Perfusion vs. oxygen delivery in transfusion with 'fresh' and 'old' red blood cells: the experimental evidence. Transfus Apheresis Sci 43: 69-78, 2010

24. Dominici M, Le Blanc K, Mueller I, Slaper-Cortenbach I, Marini F, Krause D, Deans R, Keating A, Prockop Dj and Horwitz E: Minimal criteria for defining multipotent mesenchymal stromal cells. The International Society for Cellular Therapy position statement. Cytotherapy 8: 315-317, 2006.

25. Orlic D, Kajstura J, Chimenti S, Jakoniuk I, Anderson SM, Li B, Pickel J, McKay R, Nadal-Ginard B, Bodine DM, et al: Bone marrow cells regenerate infarcted myocardium. Nature 410 : 701-705, 2001.

26. Wu KH, Han ZC, Mo XM and Zhou B: Cell delivery in cardiac regenerative therapy. Ageing Res Rev 11: 32-40, 2012.

27. Barbash IM, Chouraqui P, Baron J, Feinberg MS, Etzion S, Tessone A, Miller L, Guetta E, Zipori D, Kedes LH, et al: Systemic delivery of bone marrow-derived mesenchymal stem cells to the infarcted myocardium: feasibility, cell migration, and body distribution. Circulation 108: 863-868, 2003.

28. Zhang H, Fang J, Su H, Yang M, Lai W, Mai Y and Wu Y: Bone marrow mesenchymal stem cells attenuate lung inflammation of hyperoxic newborn rats. Pediatr Transplant 16: 589-598, 2012 .

29. Molina EJ, Palma J, Gupta D, Torres D, Gaughan JP, Houser S and Macha M: Improvement in hemodynamic performance, exercise capacity, inflammatory profile, and left ventricular reverse remodeling after intracoronary delivery of mesenchymal stem cells in an experimental model of pressure overload hypertrophy. J Thorac Cardiovasc Surg 135: 292-299, 299.e1, 2008.

30. Freyman T, Polin G, Osman H, Crary J, Lu M, Cheng L, Palasis M and Wilensky RL: A quantitative, randomized study evaluating three methods of mesenchymal stem cell delivery following myocardial infarction. Eur Heart J 27: 1114-1122, 2006. 